2 Neuberger JS, Harris J-A, Kundin WD, Bischone A, Chin TDY. Incidence of needlestick injuries in hospital personnel: implications for prevention. Am $\mathcal{F}$ Infect Control 1984;12:171-6.

3 Department of Health and Social Security. Disposal of hypodermic syringes, needles, scalpe blades etc. Health Equipment Information 1985;149:20.

4 Tye J. Needlestick injuries. Br Med $\mathcal{J}$ 1985;291:827.

5 Scottish Home and Health Department. Hazard warming. Edinburgh: SHHD, 1986. (CSA(SD)HAZ 2/86.)

(Accepted 24 April 1987)

Department of Nuclear Medicine, Glasgow Royal Infirmary, Glasgow G4 0SF R G BESSENT, MA, DPHIL, principal physicist

West of Scotland Health Boards, Department of Clinical Physics and BioEngineering, Glasgow G4 9LF

R DONNET, TENG, principal medical physics technician

A SHAW, BSC, CENG, top grade physicist

Correspondence to: $\mathrm{Mr}$ Shaw.

\section{Right atrial thrombus: a complication of total parenteral nutrition in an adult}

Complications of total parenteral nutrition include sepsis, thrombosis, and metabolic abnormalities. Although several cases of right atrial thrombosis as a complication of total parenteral nutrition have been described in premature infants, ${ }^{1-4}$ it has not, to our knowledge, been described in adults. We report such a case in an adult.

\section{Case report}

A 17 year old youth was admitted with a suspected infection of his central venous feeding line. From June 1983 he had received home parenteral nutrition after resection of virtually all his small intestine because of gangrene caused by a volvulus. He had been well until two days before his admission, when he developed a fever and rigors. On examination he was feverish with a temperature of $38^{\circ} \mathrm{C}$. His heart sounds were normal and his chest clear. Blood cultures grew Staphylococcus epidermidis and he was treated with flucloxacillin. His fever resolved and he restarted intravenous feeding.

He was readmitted two weeks later with a sudden onset of dyspnoea, fever, and dry cough. On examination he was feverish with a temperature of $39 \cdot 7^{\circ} \mathrm{C}$. His pulse rate was $130 / \mathrm{min}$ and his heart sounds were normal. He had bilateral basal crepitations. An electrocardiogram showed sinus tachycardia and a chest $x$ ray film showed a decreased vascularity in the right, mid, and lower zones. A perfusion-ventilation scan showed a perfusion defect in the right lower zone that was suggestive of a pulmonary embolus. His feeding line was removed and two

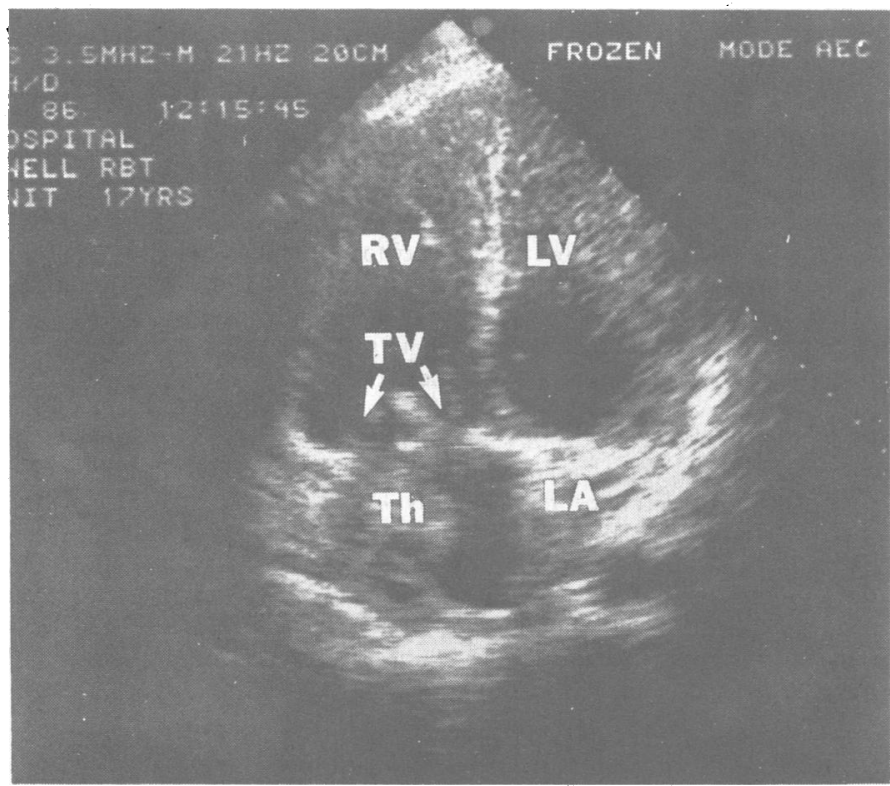

Two dimensional echocardiogram: apical view of four chambers showing thrombus $(T h)$ in right atrium. $R V=$ right ventricle, $L V=$ left ventricle, $L A=$ left atrium, and $T V=$ tricuspid valve. dimensional echocardiography was performed. It showed a large mass in the right atrium (figure) attached to the base of the atrium with a long tail protruding intermittently through the tricuspid valve into the right ventricle. The right atrium and ventricle were moderately dilated. Blood cultures grew Staph epidermidis and he was treated with antibiotics and heparin.

He was referred for surgery. A right sternotomy was performed, which showed that the right side of the heart was moderately dilated. In the right atrium was a fairly large clot attached with a wide base to the right atrial wall. It had a long tail with a bulbous end protruding through the tricuspid valve into the right ventricle. Most of the right atrial wall was thickened. The clot was excised together with a large section of the right atrial wall. The wall was repaired with a patch of bovine pericardium. Histological examination showed that the mass was composed of fibrin, and no organisms were grown. He made an excellent recovery. The antibiotics were discontinued and he was given oral anticoagulation treatment. A Broviac feeding line was inserted and at the time of writing he was continuing with total parenteral feeding.

\section{Comment}

Although the exact incidence of right atrial thrombosis as a complication of total parenteral nutrition is not known, several cases have been reported in premature infants. Pliam et al described the first case after prolonged central venous feeding in a premature infant. ${ }^{1}$ Since then 10 additional cases have been reported, all in premature infants $\mathrm{s}^{2-4}$; most presented with features of superior vena caval obstruction..$^{23}$

Our patient presented with clinical features of pulmonary embolus and suspected sepsis of his catheter. Right atrial thrombus was diagnosed from clinical presentation, a ventilation-perfusion scan, and an echocardiogram without the need for a ventriculoangiogram. Recently Wolfe et al studied 1617 patients, none of whom were infants and $95 \%$ of whom were adults. Not one case of clinically manifested pulmonary embolus was identified, and right atrial thrombus was not reported. ${ }^{5}$ Right atrial thrombi secondary to cardiac disease have been reported in adults, ${ }^{4}$ but to our knowledge ours is the first case of right atrial thrombus complicating total parenteral nutrition in an adult.

Surveillance of central venous catheters with two dimensional echocardiography should be a priority for detecting thrombi or vegetations; early diagnosis of intracardiac thrombi may prevent massive pulmonary emboli.

We thank Mr Ali N Rahman for his help and Ms J Rostron for her secretarial help.

1 Pliam MB, McGough EC, Nixon GW, Ruttenberg HD. Right atrial ball-valve thrombus: a complication of central venous alimentation in an infant. F Thorac Cardiovasc Surg 1979;78: 579-82.

2 Bucciarelli RL, Jaffe R. Non-invasive evaluation of superior vena caval and right atrial thrombosis complicating central hyperalimentation. Clin Pediatr 1983;22:302-3.

Graham L Jr, Gumbinen $\mathrm{CH}$. Right atrial thrombus and superior vena cava syndrome in a child. Pediatrics 1984;73:225-9.

4 Felner JM, Churchwell AL, Murphy DA. Right atrial thromboemboli: clinical, echocardiographic and pathophysiologic manifestations. $I$ Am Coll Cardiol 1984;4:1041-51.

5 Wolfe BM, Ryder MA, Nishikawa RA, Halsted CH, Schmidt BF. Complications of parenteral nutrition. Am F Surg 1986;152:93-9.

(Accepted 24 April 1987)

Departments of Thoracic Medicine and Surgery, University of Manchester School of Medicine, Hope Hospital, Salford M6 8HD

H CHAMSI-PASHA, MD, MRCP, research fellow

M H IRVING, CHM, FRCS, professor of surgery

Correspondence and requests for reprints to: Dr Chamsi-Pasha.

\section{National survey of use of the Angelchik antireflux prosthesis}

The Angelchik antireflux prosthesis is a silicone prosthetic implant shaped like a horseshoe that is tied around the distal oesophagus beneath the diaphragm. It has been used in the United Kingdom for a little over five years. Its insertion has been claimed to be technically uncomplicated and associated with few complications, ${ }^{1}$ and it has been reported to achieve good control of reflux. ${ }^{2}$ Several serious complications have been reported, ${ }^{3-5}$ but only a small number of patients have been studied so it is difficult to assess the true incidence of complications. The aim of this study was to investigate the experience of general surgeons using the Angelchik antireflux prosthesis in the United Kingdom and to determine the incidence and nature of serious complications and the rate of removal. 